\title{
El impuesto a los consumos especiales (ICE) aplicado a las bebidas alcohólicas incluida la cerveza.
}

\section{The special consumption tax (ICE) applied to alcoholic beverages including beer.}

Fanny Graciela Egas Moreno. ${ }^{1}$, Luz Marina Cifuentes. ${ }^{2}$, Domitilo Toala Preciado. ${ }^{3}$, Proaño Gonzalez Esther Angélica. ${ }^{4}$

DOI: https://doi.org/10.33262/visionariodigital.v3i2.477

\section{Resumen.}

El impuesto a los consumos especiales (ice) aplicado a las bebidas Alcohólicas incluida la cerveza en el Ecuador, es una investigación de enfoque cuantitativo, de alcance correlacional y de corte longitudinal que partió desde la identificación del problema de estudio y la situación actual según la reforma del 01 de Mayo del 2016, que aplica el Impuesto a los Consumos Especiales(ICE) a las Bebidas Alcohólicas, en base a la información se definieron claramente los objetivos tanto generales y específicos, por lo tanto el estado del arte ayudo afianzar los resultados que engloban características y conceptos relacionados con el tema de estudio y de investigación. Para su desarrollo utilizamos el método deductivo y analítico que se aplicó con énfasis al reconocimiento de la problemática y el conocimiento general de las variables el tipo de investigación fue descriptiva y de campo mientras el diseño fue no experimental. La población y la muestra fueron seleccionadas de la matriz de contribuyentes autorizados parala comercialización de bebidas alcohólicas, la recolección de datos se realizó mediante una encuesta que permitieron definir las respectivas conclusiones y recomendaciones cumpliendo con los objetivos de estudio que permitieron elaborar y determinar cómo incide la reforma Tributaria (ICE) en los impuestos a los consumos especiales en las bebidas alcohólicas.

Palabras claves: Impuesto, Reforma administrativa, alcoholismo, Contabilidad Nacional

\footnotetext{
${ }^{1}$ Universidad Técnica Luis Vargas Torres de Esmeraldas, Esmeraldas Ecuador, fanny.egas@utelvt.edu.ec

${ }^{2}$ Universidad Técnica Luis Vargas Torres de Esmeraldas, Esmeraldas Ecuador, luz.cifuentes@utelvt.edu.ec

${ }^{3}$ Universidad Técnica Luis Vargas Torres de Esmeraldas, Esmeraldas Ecuador, domitil2009@hotmail.com

${ }^{4}$ Universidad Técnica Luis Vargas Torres de Esmeraldas, Esmeraldas Ecuador, esther.proano@utelvt.edu.ec
} 


\section{Asbtract}

The tax on special consumption (ice) applied to alcoholic beverages including beer in Ecuador, is a quantitative approach research, correlational scope and longitudinal cut that started from the identification of the study problem and the current situation according to the reform of May 01, 2016, which applies the Special Consumption Tax (ICE) to alcoholic beverages, based on the information, the general and specific objectives were clearly defined, therefore the state of the art helped to consolidate the results which encompass characteristics and concepts related to the subject of study and research. For its development we used the deductive and analytical method that was applied with emphasis to the recognition of the problem and the general knowledge of the variables. The type of research was descriptive and field while the design was non-experimental. The population and sample were selected from the matrix of authorized taxpayers for the commercialization of alcoholic beverages, data collection was performed through a survey that allowed to define the respective conclusions and recommendations fulfilling the study objectives that allowed to elaborate and determine how the Tax Reform (ICE) affects taxes on special consumption in alcoholic beverages.

Keywords: Tax, Administrative reform, alcoholism, National Accounts

\section{Introducción}

Ecuador ocupa el sexto lugar a nivel de Latinoamérica en el consumo de alcohol por lo cual el gobierno con otros organismos de control tanto nacionales como extranjeros plantea medidas para disminuir y el excesivo consumo de alcohol, como medida de prevención ante las excesivas muertes que causa por ingerir bebidas alcohólicas (Servicios de Rentas Internas , 2018). A partir del 01 de mayo de 2016, está vigente la nueva tarifa específica de bebidas alcohólicas incluida la cerveza establecida por el Servicio de Retas Internas en las cuales se encuentran nuevas tarifas específicas y AD VALOREM para el cálculo del (ICE), la misma que se encuentra establecida en la Ley Orgánica para el Equilibrio de las Finanzas Públicas, publicada en el Suplemento del Registro Oficial 744 del 29 de abril de 2016 (Servicios de Rentas Internas , 2018). El análisis de este problema socio/económico pretende evidenciar que ha ocasionado que en la ciudad de Riobamba incremente su consumo de alcohol, lo cual debió disminuir. En otras naciones y en nuestro país, el gobierno obliga a vender bebidas alcohólicas a mayores de 18 años, no vender los días domingos y definir un horario de atención donde se expendan toda clase de bebidas alcoholizadas, lo que no es suficiente para disminuir el consumo masivo. Sin embargo, no podemos dejar de notar los ingresos que este impuesto (ICE) ha generado para el país, en lo que tiene ver recaudación de impuesto. (Córdova , 2013)

El Servicio de Rentas Internas (SRI) y Servicio Nacional de Aduanas del Ecuador (SENAE) son las principales entidades reguladoras de la percepción de este impuesto cada año analizan puntos a favor y en contra de la recaudación de todos tributos que se recaudan en el Ecuador 
como son; mensuales, semestral y anualmente (SRI, 2018). La falta de cultura tributaria y la irresponsabilidad en el cumplimiento por parte de muchos contribuyentes en la declaración de impuestos y retraso de los mismos, originan multas e intereses sobre dichos pagos, lo que no genera más ingresos. (Zabala , 2015)

Los impuestos en el Ecuador tienen como objetivo principal la distribución de los recursos necesarios y equitativos para cubrir los gastos que debe hacer el Estado anualmente mediante el Presupuesto General del Estado (PGE), existen dos principales tipos de impuestos; los directos son los que recaen sobre las personas o entidades y gravan directamente la capacidad económica de las personas, mientras que los impuestos indirectos grava el consumo, no afecta de manera directa los ingresos de un contribuyente sino que recae sobre el costo del consumidor final (Sierra , 2015). Bajo estos antecedentes la presente investigación se encuentra basada en un estudio y análisis del Impuesto a los Consumos Especiales (ICE) a las bebidas alcohólicas incluida la cerveza como producto específico, mediante la última reforma del 01 de Mayo del 2016 establecida en la Ley Orgánica para el Equilibrio de las Finanzas Públicas, publicada en el Suplemento del Registro Oficial 744 del 29 de abril de 2016 (Asamblea Nacional Del Ecuador , 2008). Determinar cuál fue su impacto de efectividad y evolución en la recaudación tributaria en el cantón Riobamba y así describir, analizar los posibles efectos económicos y sociales que causa el impuesto, y conocer como el gobierno toma medidas por el excesivo consumo de alcohol, la metodología que se utilizó se basa en una investigación descriptiva y diseño no experimental, analítica, explicativa.

Mediante la aplicación del sistema tributario y sus entes reguladores para los impuestos a los consumos especiales es necesario comprender y conocer la base legal en la que se maneja; en el marco teórico especificando, a quien afecta directa o indirectamente este impuesto, al consumidor final como principal objeto de investigación, finalmente la información analizada y recopilada servirá para determinar las conclusiones y recomendaciones en base a los resultados descritos anteriormente (Santillan, 2015).

El presente tema elegí es para saber y conocer cómo influye la nueva resolución emitida el 04 de mayo del 2016 y niveles máximos de ingresos y consumo de alcohol en la ciudad de Riobamba y que medidas preventivas el Gobierno ha tomado para controlar el excesivo consumo de alcohol. S

e ha comprobado que el consumo de bebidas alcohólicas incremento en el Ecuador según las dos últimas reformas tributarias, lo que sobrelleva a muertes y enfermedades catastróficas para la sociedad, por el consumo de este producto es una de las causas que contribuye negativamente a la salud de los consumidores igualmente positivamente a la recaudación de impuestos que favorece al estado ecuatoriano. Con el propósito de calmar o contrarrestar el consumo de bebidas alcohólicas se implementó el impuesto a los consumos especiales, la relación obtenida ha sido inversa ya que su finalidad cumplió otra al mismo tiempo, en sentidos opuestos. 
Este trabajo logra demostrar los resultados obtenidos relacionados al impacto económico y social que ha marcado el impuesto a los consumos especiales en la comercialización de bebidas alcohólicas. El análisis del impacto económico social del impuesto a los consumos especiales en la comercialización de bebidas alcohólicas en la ciudad, podremos llegar a entender que la aplicación de las medidas que aplique el estado tiene efecto en la ciudadanía.

Mediante este análisis nos permitirá conocer minuciosamente el impacto que ha sufrido la economía ante las nuevas reformas tributarias en los consumidores de Bebidas alcohólicas.

\section{Método y materiales}

La metodología para realizar este análisis, será descriptiva y de enfoque cuantitativo, de alcance correlacional y de corte longitudinal la cual permitió obtener información confiable de fuentes primarias y secundarias mediante cuadros comparativos y para las encuestas con la recolección, depuración y validación de los datos e información obtenida mediante los diferentes estudios previamente elaborados.

Donde se aplicarán los siguientes métodos como es el deductivo, se aplicará en la comprensión de conceptos, principios, definiciones, leyes y normas generales que rodean a la Investigación el Impuesto a los Consumos Especiales (ICE) aplicado a las bebidas alcohólicas además el método analítico se aplicará con énfasis en el reconocimiento de la problemática en el Ecuador, así como en la determinación de conclusiones y recomendaciones en base a los resultados obtenidos.

El diseño de la Investigación para obtener la información de la investigación es no experimental ya que no se van a manipular las variables y de enfoque cualitativo y de tipo de investigación de campo ya que se trabajar directamente con los expendedores y comercializadores de bebidas alcohólicas.

Población La población de la investigación constituye 315 expendedores autorizados por el Servicio de Rentas Internas (SRI) que se encuentran distribuidos en las parroquias de la ciudad de Riobamba para la venta y comercialización de bebidas alcohólicas hasta este año. Para el estudio se trabajará con encuesta. quien será dirigida a los representantes o dueños de las principales expendedoras y embotelladoras de un sector a los expendedores

\section{Análisis de resultado}

Esta vigente la nueva tarifa específica de bebidas alcohólicas incluida la cerveza, en las cuales se encuentran bebidas alcohólicas, cerveza artesanal e industrial y las nuevas tarifas específicas y valore para el cálculo del (ICE), la misma está establecida en la Ley Orgánica para el Equilibrio de las Finanzas Públicas, publicada en el Suplemento del Registro Oficial 744 del 29 de abril de 2016. 
Tabla 1. Productos Marcados A Nivel Nacional

\begin{tabular}{cc}
\hline Producto & Productos Marcados A Nivel Nacional \\
\hline Cerveza & $1.971,414$ millones \\
Bebidas alcohólicas & 88,121 millones \\
Cigarrillos & 115,309 millones \\
& Total \\
\hline
\end{tabular}

Elaborado por: Grupo investigador

Es un sistema de identificación, marcación, autentificación, rastreo y trazabilidad fiscal que se aplicará a cigarrillos, cervezas y bebidas alcohólicas de fabricación nacional. Este servicio brindará una solución integral para obtener información respecto de la producción, comercialización y aspectos de interés tributario de los mencionados productos gravados con el Impuesto a los Consumos Especiales ICE. El Convenio Marco para el Control del Tabaco de la Organización Mundial de la Salud (CMCT), fue firmado por Ecuador en marzo de 2004 y ratificado en julio de 2006. Su artículo 15 señala que es necesaria la eliminación de todas las formas de comercio ilícito de productos de tabaco (contrabando, fabricación ilícita y falsificación).

El 22 de julio de 2011 se publica en Ecuador, la Ley Orgánica para la Regulación y Control del Tabaco mediante el Registro Oficial Nro. 497, en el cual se establece las directrices para efectuar el control del tabaco.

El Protocolo para la eliminación del Comercio ilícito de productos del Tabaco, fue suscrito por Ecuador en el año 2013 y ratificado en junio de 2015. En su artículo 8, señala que cada parte establecerá bajo su control, un sistema de seguimiento y localización de todos los productos de tabaco que se fabriquen o importen en su territorio.

Tabla 2. ¿Sabe usted que existe reformas tributarias para bebidas alcohólicas?

\begin{tabular}{ccc}
\hline DATOS & FRECUENCIA & PORCENTAJE \\
\hline SI & 15 & $17 \%$ \\
NO & 75 & $83 \%$ \\
\hline
\end{tabular}

Elaborado por: Grupo investigador 
Del total de 90 encuestados referentes al $100 \%$ podemos observar que 75 de los comercializadores de bebidas alcohólicas no saben o no tiene conocimiento de la existencia de reformas tributarias dando como resultado un 83\%, y que 15 de comercializadores encuestados si tiene conocimiento proporcionando el $17 \%$ de la población encuestada.

Tabla 3. ¿Usted conoce lo que compone el ICE en bebidas alcohólicas para su comercialización?

\begin{tabular}{ccc}
\hline DATOS & FRECUENCIA & PORCENTAJE \\
\hline SI & 22 & $24 \%$ \\
NO & 68 & $76 \%$ \\
\hline
\end{tabular}

Elaborado por: Grupo investigador

De los 90 expendedores y comercializadores de bebidas alcohólicas entrevistados el $24 \%$ afirma que si tiene conocimiento como está compuesto el Ice para su comercialización, mientras que el $76 \%$ de la población restante tiene un desconocimiento.

Tabla 4. ¿Cree que el Impuesto (ICE) disminuyo el consumo excesivo de bebidas alcohólicas?

\begin{tabular}{ccc}
\hline DATOS & FRECUENCIA & PORCENTAJE \\
\hline SI & 7 & $8 \%$ \\
NO & 83 & $92 \%$ \\
\hline
\end{tabular}

Elaborado por: Grupo investigador

De los resultados obtenidos se desprende 7 expendedores entrevistados sobre un total de 90 que si piensa que el Impuesto a los Consumos Especiales disminuyo el consumo excesivo de bebidas alcohólicas en el Ecuador, por otra parte 83 expendedores y comercializadores piensan que no disminuyo el consumo de alcohol dando un total del $92 \%$ del total de la población entrevistada.

Tabla 5. ¿Cree que la última reforma tributaria actualizada en el 2016 aumento la recaudación de los tributos para el presupuesto General del Estado?

\begin{tabular}{lcc}
\hline DATOS & FRECUENCIA & PORCENTAJE \\
\hline SI & 51 & $43 \%$ \\
NO & 39 & $57 \%$ \\
\hline
\end{tabular}

Elaborado por: Grupo investigador 
Se puede observar que el total de 90 encuestados, 51 afirman que la última reforma tributaria emitida en el 2016 aumento la recaudación de los tributos para el Presupuesto General del Estado dando un $43 \%$ y los 39 restantes consideran que no hubo un incremento de recaudación dando un total del $57 \%$ sobre el $100 \%$.

Tabla 6. ¿Dejo de vender y comprar bebidas alcohólicas por el alza del impuesto (ICE) a las bebidas alcohólicas

\begin{tabular}{rcc}
\hline DATOS & FRECUENCIA & PORCENTAJE \\
\hline SI & 21 & $23 \%$ \\
NO & 69 & $77 \%$ \\
\hline
\end{tabular}

Elaborado por: Grupo investigador

Mediante la encuesta realizada se pudo determinar que 21 de los comercializadores y expendedores de bebidas alcohólicas en el Ecuador si dejo de vender y comprar por el alza del Ice dando un $23 \%$, mientras que el $77 \%$ considera q no afecto sus ventas y compras siendo las 69 comercializadores restantes.

Tabla 7. ¿De las siguientes bebidas alcohólicas incluida la cerveza cual es la que tuvo mayor demanda de consumo?

\begin{tabular}{ccc}
\hline DATOS & FRECUENCIA & PORCENTAJE \\
\hline Cerveza & 58 & $64 \%$ \\
Vino & 2 & $2 \%$ \\
Wisky & 17 & $19 \%$ \\
Ron & 8 & $9 \%$ \\
Tequila & 5 & $6 \%$ \\
Otra & 0 & $0 \%$ \\
\hline
\end{tabular}

Elaborado por: Grupo investigador

A través de la encuesta realizada para conocer cuál es la bebida que tiene mayor demanda de consumo en la ciudad de Riobamba en el año 2016 nos supieron manifestar 58 encuestados que es la cerveza tiene mayor demanda de consumo dando un $68 \%$, seguido del wisky siendo un $19 \%$, el ron con el $9 \%$, seguido del tequila con el $6 \%$ y por último tenemos al vino con un $2 \%$ dando un total del $100 \%$ de la población encuestada. 
Tabla 8. ¿Cuál del siguiente listado de bebidas alcohólicas incluida la cerveza le genero más ingresos económicos al establecimiento?

\begin{tabular}{ccc}
\hline DATOS & FRECUENCIA & PORCENTAJE \\
\hline Cerveza & 52 & $58 \%$ \\
Vino & 0 & $0 \%$ \\
Wisky & 20 & $22 \%$ \\
Ron & 7 & $8 \%$ \\
Tequila & 10 & $11 \%$ \\
Otra & 1 & $1 \%$ \\
\hline
\end{tabular}

Elaborado por: Grupo investigador

La encuesta realizada para conocer cuál es la bebida alcohólica que le genera más ingresos económicos al establecimiento manifestaron que la cerveza con un $58 \%$ es la que les genera más ingresos, mientras que el wisky cuenta con el $22 \%$, el tequila el $11 \%$, el ron cuenta con el $8 \%$, y por ultimo tenemos otras bebidas que 1 establecimiento manifestó que el zhumir le generaba más ingresos económicos con el 1\%, dando un total del 100\% de la población entrevistada.

Tabla 9. ¿Está de acuerdo que el gobierno disponga de tarifas específicas mediante reformas tributarias para las bebidas alcohólicas?

\begin{tabular}{ccc}
\hline DATOS & FRECUENCIA & PORCENTAJE \\
\hline SI & 46 & $51 \%$ \\
NO & 44 & $49 \%$ \\
\hline
\end{tabular}

Elaborado por: Grupo investigador

Al realizarla encuesta al referirnos si están de acuerdo que el gobierno disponga de tarifas específicas para bebidas alcohólicas 46 comercializadores nos supieron que, si están de acuerdo el $51 \%$, mientras que 44 encuestados manifestaron que no están de acuerdo dando el $49 \%$ restante.

\section{Discusión}

Se pudo observar que existe una falta de información por parte de los comercializadores y expendedores de bebidas alcohólicas en el Ecuador, al momento de vender sus productos un $82 \%$ de comercializadores desconoce que existen reformas tributarias en bebidas alcohólicas, 
de la misma manera existe una escasa cultura tributaria, esto implica una consecuencia como poner precios variados al momento de ser expendidos, de la misma manera desconoce cómo está compuesto el ICE en sus productos al momento de ser comercializados.

En cuanto a la interrogante sobre si piensan que el Impuesto a los Consumos Especiales (ICE) disminuyo el consumo excesivo de bebidas alcohólicas y la nueva reforma que fue emitida el 01 de Mayo del 2016 aumento la recaudación de los tributos en el Ecuador, en relación a los resultados obtenidos son que el consumo de bebidas alcohólicas va en aumento y representa el $57 \%$ y manifiestan que las personas no les importa pagar un valor adicional o extra por tomar una bebida alcohólica, y en relación si aumento la recaudación de los tributos ellos si piensan que mediante reformas el estado lograra recaudar mayores ingresos de impuestos y esto incrementará el presupuesto General del Estado para el próximo periodo. Con un $64 \%$ del total de encuestados manifestaron que la cerveza tiene mayor demanda de consumo en el Ecuador ya que es una bebida tradicional y que se la toma a cualquier hora del día ya que no es una bebida muy fuerte como el wisky, ron, tequila, aguardiente etc. De la misma manera el $58 \%$ manifestó la cerveza es la que le genera mayores ingresos económicos al establecimiento al momento de su comercialización. Las personas expendedoras y comercializadores manifestaron que están de acuerdo que el gobierno promulgue y disponga tarifas especificas mediante reformas tributarias para la comercialización de bebidas alcohólicas ya que esto les ayuda para tener precios fijos entre todos los comercializadores en tiendas, bares, restaurants, con un $51 \%$ del total de encuestados.

\section{Conclusiones}

- El incremento de los impuestos en los consumos especiales, es una medida que ha ayudado a mantener el presupuesto de nuestro país, sin embargo, no ha podido cumplir con el objetivo 30 primordial, ir reduciendo el consumo de bebidas alcohólicas. Los niveles de recaudación por el Impuesto a los consumos especiales (ICE) aumentó un $5.58 \%$ en relación a los años 2015 y esto hace referencia que si influye satisfactoriamente la aplicación de la nueva reforma tributaria. La bebida alcohólica que tiene mayor demanda de consumo y de ingresos económicos en los establecimientos autorizados por el Servicio de Rentas Internas (SRI) en el Ecuador es la cerveza, seguido del wiski y del tequila.

\section{Referencias Bibliografías}

Asamblea Nacional Del Ecuador . (20 de mayo de 2008). Constitución de la República del Ecuador. Obtenido de www.oas.org: https://www.oas.org/juridico/mla/sp/ecu/sp_ecu-int-text-const.pdf 
Córdova , A. (2013). Análisis del impuesto a consumos especiales en el comercio exterior de whisky en el ecuador, y su incidencia en el comercio exterior ecuatoriano, en el periodo 2008-2011. Tesis pre grado UNiversidad Tecnológica Equinoccial. Quito, Ecuador . Obtenido de http://repositorio.ute.edu.ec/bitstream/123456789/8269/1/52728_1.pdf

Hurtado , M., \& Rodriguez , E. (2 de marzo de 2010). Impuesto al consumo especiales: Base imponibles. Tesis de pre grado, Univerisdad de Cuenca, 41. Cuenca, Azuay, Ecuador. Obtenido de http://dspace.ucuenca.edu.ec/jspui/bitstream/123456789/1421/1/tcon449.pdf

Santillan, J. (2015). Sistemas de Control Interno (Tercera edición ed.). México. Obtenido de

http://www.pearsonenespanol.com/docs/librariesprovider5/santillana/97860732311 90_santillana_resolucion_pdf51e66e8b436366b1aea8ff00004a2a88.pdf

Servicios de Rentas Internas . (12 de abril de 2018). Fiscalia Internacional . Obtenido de http://www.sri.gob.ec/web/guest/fiscalidad-internacional2

Sierra , P. (15 de abril de 2015). Diseño e implementación de un manual de control interno para la asociación de ganaderos del Altiplano Cundiboyacense y Occidente De Boyaca “Asogaboy". Tesis de pregrado Universidad Pedagógica y Tecnológica de Colombia. Bogota. Obtenido de https://repositorio.uptc.edu.co/bitstream/001/2047/1/TGT-658.pdf

SRI. (2018). Sistema de identificación, marcación, autentificación, rastreo y trazabilidad fiscal de bebidas alcohólicas, cervezas y cigarrillos de producción nacional. Obtenido de SIMAR: http://www.sri.gob.ec/web/guest/simar

Zabala , R. (9 de febrero de 2015). El impuesto a los consumos especiales (ICE) aplicado a las bebidas alcohólicas en Ecuador, caso: cervezas (2008-2013). Tesis de pos grado Universidad de Guayaquil Facultad de Ciencias Económicas, 96. Guayaquil, Guayas, Ecuador . Obtenido de http://repositorio.ug.edu.ec/bitstream/redug/7036/1/TESIS\%20ROXANA\%20ZAV ALA\%20.pdf

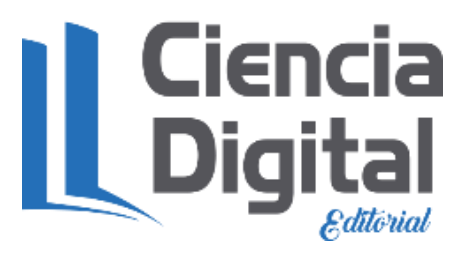


Para citar el artículo indexado.

Egas F., Cifuentes L., Toala D., Proaño E. (2019). El impuesto a los consumos especiales (ICE) aplicado a las bebidas alcohólicas incluida la cerveza., revista electrónica Visionario Digital 3(2), 294-304. Recuperado desde: http://www.visionariodigital.org

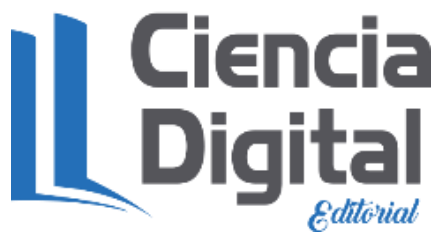

El artículo que se publica es de exclusiva responsabilidad de los autores y no necesariamente reflejan el pensamiento de la Revista Ciencia Digital.

El articulo queda en propiedad de la revista y, por tanto, su publicación parcial y/o total en otro medio tiene que ser autorizado por el director de la Revista Ciencia Digital.
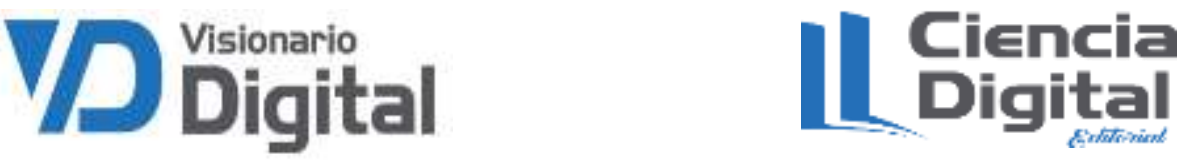